\title{
Host Modulatory Therapy: A Novel Approach in Periodontal Therapy
}

\author{
Dr. Raju Anarthe ${ }^{1}$, Dr. Ameet Mani ${ }^{2}$, Dr. P.P. Marawar ${ }^{3}$ \\ 1: Sr. Lecturer, 2-Reader, 3-Professor and Head \\ Dept. of Periodontology, Rural Dental College, Pravara Institute of Medical Sciences, Loni- 413736 \\ Maharashtra State, India
}

\begin{abstract}
Although bacterial plaque is primary etiologic factor in the initiation and progression of periodontal diseases, the host's reactions to the presence of the bacteria are the one which mediates the periodontal tissue destruction. The periodontal tissue destruction is believed to be due to the host response, it is logical to consider therapeutic approaches that modulate the host response in addition to antibacterial and analgesic approaches in the management of periodontal diseases. A number of host modulatory agents like chemically modified tetracyclines (CMT), non-steroidal anti-inflammatory drugs, and bisphosphonates are effective in treating periodontal diseases in adjunct to the nonsurgical and surgical periodontal therapy. This paper reviews the Host Modulatory Therapy which is the effective means when used as an adjunct to mechanical periodontal therapy in treating periodontal diseases.
\end{abstract}

Key Words: Bisphosphonates, Chemically modified tetracyclines, Host modulation, Non-steroidal antiinflammatory drugs, periodontal disease.

\section{INTRODUCTION}

Periodontitis is the disease primarily affecting the supporting structures of teeth, which causes clinical attachment loss due to apical shifting of junctional epithelium, mobility of teeth, gingival recession and pathological migration of teeth. Significant portion of the population is susceptible to the periodontal diseases; this occurs due to the destructive mechanis $m$ of periodontal disease. Still a number of individuals are relatively resistant to the severe forms of periodontal disease due to the protective mechanism of individual even after presence of bacterial plaque and calculus. This leads to the hypothesis that there are susceptibility factors or risk factors that modulate susceptibility or resistance of individuals to destructive periodontal diseases.

Host modulatory therapy is a new treatment modality that has been incorporated into the dental therapeutics but it has not been well implemented in the dental practice due to the easy unavailability of host modulatory agents in India. Host can be defined as "the organism from which a parasite obtains nourishment," or in the transplantation of tissue, "the individual who receives the graft" [1]. Modulation is defined as "the alteration of function or status of something in response to a stimulus or an altered chemical or physical environment"[1]. In periodontal diseases, the "host" clearly is the individual who harbors periodontal pathogens; however, it was not clear for many years whether it was possible to modulate the host response to these pathogens. Host modulation with chemotherapeutic agents or drugs is a promising new adjunctive therapeutic opportunity for the management of periodontal diseases. The concept of host modulatory therapy was first introduced to dentistry by Williams and Golub et. al. and then expanded by many other researchers in the dental profession. Golub and colleagues discussed "host modulation with tetracyclines and their chemically modified analogues"[1]." Host modulatory therapy is a treatment concept that aims to reduce tissue destruction and stabilize or even regenerate the periodontium by modifying or down regulating destructive aspects of host response and up regulating protective or regenerative responses by means of pharmacological agents. [2].

The aim of this review is to understand this new era in periodontal disease management by using host modulatory therapy

\section{DISCUSSION}

Concepts of the etiology of periodontal disease have changed noticeably in the last four decades. In 1985 research began to focus on bacterial-host interactions. Several specific subgingival oral bacteria including porphyromonus gingivalis, actinobacillus aggregatibater, prevotela intermedia, bacteroides forsythus and perhaps others such as campylobacter rectus, fusobacterium nucleatum, and spirochetes are associated with severe type of periodontal diseases [3]. Protective aspects of the host response include recruitment of neutrophils, production of protective antibodies, and possibly the release of antiinflammatory cytokines including transforming growth factor (TGF- $\beta$ ), interleukin-4 (IL-4), IL-10, and IL-12. Persistent bacterial aggression disrupts homeostatic mechanisms and results in release of proinflammatory cytokines (e.g., IL-1, IL-6, tumor necrosis factor- $\alpha$ (TNF- $\alpha$ ), proteases (e.g., Matrix Metallo proteinase's), and prostanoids (e.g., prostaglandin E2 [PGE2]), which can endorse extracellular matrix destruction in the periodontium and stimulate bone resorption, tooth mobility and tooth loss. It is now clear that host factors play a major role in the etiopathogenesis of periodontal diseases [4]. Researchers are also investigating host modulatory therapies which aim to modify or reduce destructive aspects of the host response so that the immune-inflammatory response to plaque is less damaging to the periodontal tissues. 
Various HMT have been developed to block or modify the pathways of periodontitis [2].

- Inhibition of matrix metalloproteinase (MMPs): This is achieved by chemically modified tetracyclines (CMTs)

- Inhibition of arachidonic acid metabolite : Through NSAIDs

a. COX-1 inhibitors: Indomethacin, Flurbiprofen, Naproxen.

b. COX-2 inhibitors: Rofecoxib.

c. COX and LOX inhibitors: Triclosan, Topical ketoprofen.

d. LOX inhibitors: Lipoxins.

- Modulation of bone metabolism

a. Bisphosphonates

b. Harmone replacement therapy (HRT)

c. Calcium supplementation.

- Regulation of immune and inflammatory response:

a. Suppressing proinflammatory cytokines: IL1 and TNF- $\alpha$ receptor antagonist.

b. Nitric oxide inhibition.

c. Generation of protective antibodies through vaccination.

d. Infusion/ supplementary anti-inflammatory cytokines: IL-4 and IL-10.

Inhibition of matrix metalloproteinase (MMPs): Metallo proteinases (MMPs) are group of proteolytic enzymes produced by fibroblasts, keratinocytes, macrophages, neutrophils and endothelial cells which causes alveolar bone destruction in periodontal diseases. In pathological conditions, macrophage derived tumor necrosis factor- $\alpha$, interleukin-1 $\beta$ and interleukin-6 markedly increases the local production of various matrix metallo proteinases in periodontal tissues. Collagenases, gelatinases and metallo elastases are the matrix metallo proteinases present at the site of periodontal destruction.

Chemically Modified Tetracyclines (CMTs): Inhibition of matrix metalloproteinase is achieved by chemically modified tetracyclines (CMTs). Chemically modified tetracyclines (CMTs) are those which lacks dimethyl-amino group on $4^{\text {th }}$ carbon atom. However it is not the antibacterial property of CMTs but their ability to prevent connective tissue breakdown (anticollagenase activity) and bone loss (osteoclast inhibition activity) has found much of interest in host modulatory therapy. In 1985, CMTs had been discovered to have anticollagenolytic activity and was proposed as a hostmodulating agent for periodontal treatment. [5]

Long term use of antimicrobial doses is limited by potential development of resistant microorganisms and gastrointestinal distress. In an attempt to bypass this crisis while maintaining host modulatory properties researchers have evaluated the efficacy and wellbeing of subantimicrobial doses of tetracycline in conjunction with mechanical procedures in the treatment of periodontitis. Recently a formulation containing a sub antimicrobial dose of Doxycycline (SDD) (Doxycycline hyclate $20 \mathrm{mg}$; Periostat, CollaGenex, Pharmaceuticals Newton PA) is FDA approved and ADA accepted. It is indicated as an adjunct to scaling and root planing in the treatment of chronic periodontitis. The $20 \mathrm{mg}$ twice per day dose for 3 to 9 months exert its therapeutic effect by enzyme, cytokine, and osteoclast inhibition, rather than by any antibiotic effect [5].

SDD works so well as a host modulatory agent because of its pleiotropic effects on multiple components of the host responses. Recent clinical trials have focused on doxycycline because it was found to be a more effective inhibitor of collagenase than minocycline or other tetracyclines and because of its safety profile, pharmacokinetic properties, and systemic absorption [6]. In this respect, a recent study compared the efficacy of scaling with and without adjunctive SDD among patients who consistently exhibited elevated gingival Crevicular fluid collagenase levels prior to treatment. It was determined that patients who received SDD demonstrated less clinical attachment loss than individuals who received scaling alone during a 36-week period. (5)

In addition to use of SDD in host modulatory therapy, ten different chemically modified tetracyclines (CMTs) have been developed, nine of which inhibit MMPs and do not possess antimicrobial properties. CMTs have been reported to reduce the progression of experimentally induced periodontitis in animal models. The development of recombinant tissue inhibitor of matrix metalloproteinase (TIMP) and synthetic MMP inhibitors offers promising therapeutic approaches for the treatment of conditions characterized by excessive MMP activity. [7]

Mechanism of action of chemically modified tetracyclines (CMTs): [2, 8]

- Inhibition of production of epithelial derived MMPs by inhibiting cellular expression and synthesis.

- Inhibits or chelates the calcium ions that MMPs requires for their action.

- Inhibit already active MMPs.

- Scavenges reactive oxygen species.

- Modulates the osteoclast functions. 
Inhibition of arachidonic acid metabolite: Arachidonic acid can be metabolized via cyclooxgenase (COX) or lipooxygenase (LOX) pathways. The final products of the COX pathway include prostaglandins, prostacyclin, and thromboxane. Elevated levels of PGE2 and other AA metabolites have been reported in gingival crevicular fluid and periodontal tissues in patients exhibiting gingivitis, periodontitis, and periimplantitis. Mean crevicular PGE2 concentrations are also significantly elevated in patients who exhibit disease progression compared to periodontally stable individuals. One proposed approach to modulate the host response is inhibition of enzymes responsible for the release of these destructive products.

Nonsteroidal Anti-Inflammatory Drugs (NSAIDs): NSAIDs currently under investigation for host modulatory therapy are Flurbiprufen, Naproxen, Meclofenamate and Ketorolac[9]. They limit the progression of periodontitis through their ability to reduce inflammation and bone resorption [10]. The topical application of NSAIDs is an alternate method to deliver these agents. In general, topical application of NSAIDs is possible because these drugs are lipophilic and are absorbed into gingival tissues. NSAIDs that have been evaluated for topical application include ketorolac tromethamine rinse and S-ketoprofen dentifrice [2]. Recently, selective NSAIDs called coxibs (COX-2 inhibitors, Nimesulide) have been developed that selectively block the isoenzyme associated with inflammation (COX-2). Clinical trials have demonstrated that use of these agents cause significantly fewer gastrointestinal adverse events than does treatment with non-selective NSAIDs.

Tricloson: A compound which has received importance as both an antibacterial and anti-inflammatory agent is triclosan. Triclosan (2,4,41trichloro-2-hydroxydiphenyl ether) is a non-ionic antimicrobial agent. Triclosan also inhibits Cyclooxygenase and Lipoxygenase and thus may interfere with the production of AA metabolites [2,4]. Use of a dentifrice containing sodium fluoride $(0.243 \%)$ and triclosan $(0.3 \%)$ with $2.0 \%$ PVM/MA copolymer (the non-proprietary designation for a polyvinylmethyl ether maleic acid copolymer) reduced the frequency of deep periodontal pockets and the number of sites exhibiting attachment and bone loss in patients deemed highly susceptible to periodontitis.

Modulation of bone metabolism: Severity of periodontitis is aggregated by the alveolar bone loss, osteoclasts are responsible for the bone loss. Modulation of this is achieved by bisphosphonates in HMTs.

Bisphosphonates: (Alendronate, Residronate, Zoledronic Acid, Pamidronate, Ibadronate): Bisphosphonates were introduced in 1990 for treatment of osteoporosis and osteolytic tumors. They are second group of drugs under investigation for their ability to modulate the bone loss and prevent bone resorption. They are primarily used to treat hypercalcemia, paget's disease and osteoporosis. Bisphosphonates are non-biodegradable analogs of pyrophosphate that have a high affinity for calcium phosphate crystals and that inhibit osteoclast activity. Bisphosphonates bind to and accumulate in bone and remain there for months. They inhibit osteoclast attachment to bone, induce apoptosis of osteoclasts, and inhibit differentiation of bone marrow precursor cells into osteoclasts, thus contributing to inhibition of bone resorption and increased bone mass. These compounds also appear to inhibit matrix metalloproteinase activity through a mechanism that involves chelation of cations. [11]

One of these drugs, alendronate has been evaluated in ligature induced periodontitis models and assessed for changes in bone density. Alendronate inhibited the loss of bone density in these models. However minimal effects were demonstrated on clinical parameters. A pilot human clinical study was performed to assess the efficacy of alendronate in slowing alveolar bone loss associated with periodontitis. Limitations of these drugs on prolonged use may lead to inhibition of bone mineralization and subsequent osteomalacia, change in white blood cell counts and jaw necrosis. However newer generation of bisphophonates appear to minimize this activity. Currently they are still under investigation and may be soon available for treatment of periodontitis. [11]

\section{Mechanism of action of Bisphosphonate [12]}

A) Tissue level

- Decrease bone turnover due to decrease bone eruption.

- Decrease number of new bone multicellular units.

- Net positive whole body bone balance.

B) Cellular level

- Decrease osteoclast recruitment

- Increase osteoclast apoptosis

- Decrease osteoclast adhesion

- Decrease depth of resorption site

- Decrease release of cytokines by macrophages

- Increase osteoblasts differentiation and number. 
C) Molecular level

- Inhibits mevalonate pathway (can result in perturbed cell and induction of apoptosis.

- Decrease post-transitional prenylation of GTP blinding proteins.

Contra indications for use:

- Sensitivity to phosphate.

- GI upset

Drawbacks:

- Chronic administration over long periods to be effective.

- High cost and accessibility.

- A full body irradiation that would occur since these agents have to be administered IV.

Estrogen and Selective Estrogen Receptor Modulators (SERMS): Estrogen deficiency is associated with increase in bone resorption in females with osteoclast formation and activity and reduced osteoclast apoptosis. Treatment with estrogens clearly inhibit bone loss as well as bone turnover and increase bone mineral density. The estrogens inhibit both osteoclast activity and differentiation by regulating production of anti-inflammatory and pro-inflammatory cytokines by osteoblasts and monocytes. The effect of steroid hormones as metabolic mediators of the expression of cytokines may be reasonable explanation for the protective effect of estrogen supplementation against periodontal disease. The discovery of the agents able to exert full or partial estrogen effects on various tissues led to the development of a new class of drug known as SERMs. The mechanism by SERMs inhibit bone resorption is likely to be the same as estrogens mechanism, by blocking production of cytokines that promote osteoclast differentiation and by promoting osteoclast apoptosis. SERMs appear to offer many of the benefits of estrogen with minimal adverse effects. Raloxifen is the first drug in this class approved for the treatment of osteoporosis. Taxonifen is another drug of this class used in follow up treatment of some women with breast cancer [12].

Anti-integrins: An early Key event in the bone resorption process is the attachment of the osteoclast to the bone matrix. This matrix attachment is mediated by integrin primarily $\mu \mathrm{vb} 3$ and result in the intimate contact of the osteoclast with the matrix to be resorbed and formation of the sealing zone that enables the osteoclast to isolate a micro-environment beneath it to facilitate resorption. Blocking the adhesion of osteocalsts to their target matrix through the use of agents that disrupt integrins has been reported to inhibit bone resorption and may provide viable option after clinical investigation [12].

Regulation of immune and inflammatory response: Initial host responses to bacterial infections include activation and recruitment of neutrophils and macrophages. These cells subsequently release mediators including reactive oxygen species, which are antagonistic to plaque biofilms, but which in excess may initiate inflammation. NO is a highly reactive free radical reacting with metal and thiol residues leading to lipid peroxidation, protein and DNA damages and stimulation of cytokine release. An exaggerated production of NO has been implicated in the pathophysiology of several inflammatory processes such as arthritis, colitis and ileitis [13].

Animal experiments have shown that pharmacological inhibition of iNOS with mercaptoalkyl guanidines was associated with decreased inflammation, haemorrhagic shock and arthritis scores. This may be explained by the fact that this class of drugs (e.g. mercaptoethylguanidines (MEGs) is able to (i) inhibit COX (ii) scavenge peroxinitrite (i.e. the product of NO and superoxide) and (iii) block iNOS. However further studies are needed to substantiate its therapeutic effects in periodontal diseases [14].

Cytokine receptor antagonists: Components of the biofilm also encourage host cells to produce proinflammatory cytokines including IL- $1 \beta$ and TNF- $\alpha$, which can induce connective tissue and alveolar bone destruction [15]. The catabolic activities of these cytokines are controlled by endogenous inhibitors that include IL-1 and TNF receptor antagonists. When administered for therapeutic purposes, these antagonists can reduce inflammation. The use of cytokine receptor antagonists to inhibit periodontal disease progression has been investigated in a ligature induced periodontitis in non-human primate model. It was demonstrated that IL-1/TNF antagonists partially inhibited periodontal disease progression. However, the use of cytokine antagonists to treat human periodontal disease needs to be evaluated [16].

Cytokines implicated in suppression of the destructive inflammatory response include IL-4, IL-10, IL- 11, and TGF- $\beta$. Both IL-4 and IL-10 can target macrophages and inhibit the release of IL-1, TNF, reactive oxygen intermediates, and nitrous oxide. IL-4 also induces programmed cell death (apoptosis), which reduces the number of infiltrating inflammatory macrophages. It can also upregulate the production of IL-1 receptor antagonists. The evidence that IL- 4 is deficient in diseased periodontal tissues and the finding that exogenous IL-4 administration in experimental arthritis reduces inflammation, suggest that use of this cytokine may provide a therapeutic benefit in the treatment of periodontal diseases[17]. Recently, recombinant human IL-11, which inhibits production of TNF- $\alpha$, IL-1 and nitrous oxide was also shown to reduce disease progression in a ligature- induced periodontitis canine model.

Other locally administered host modulatory agents: A number of local host modulatory agents like enamel matrix derivatives (EMDs), growth factors and bone morphogenic proteins have been investigated for potential use as adjuncts to surgical therapy, not only to improve healing but also to stimulate regeneration of lost tissues, periodontal ligament, cementum and thus completely restoring complete periodontal attachment apparatus. The only local host modulatory agent approved by FDA for adjunctive use during surgery is Emdogain. [2] Other host inflammatory mediators being investigated for modulation include nuclear factor kappa B and endothelial cell adhesion molecules. However, the role of these inflammatory mediators in periodontitis needs to be evaluated. [18] 


\section{CONCLUSION}

The recognized importance of the host inflammatory response in the pathogenesis of periodontal diseases presents the opportunity to explore new treatment strategies. The adjunctive use of HMT with mechanical periodontal therapy has been reported involving non-surgical and surgical approaches. Clinical trials have shown that inhibition of AA metabolites with NSAIDs reduces gingival inflammation and periodontal disease progression. However, recently reported serious adverse effects of some COX-2 inhibitors preclude their use as an adjunct to mechanical periodontal therapy. Evidence shows that non-surgical periodontal therapy with adjunctive $20 \mathrm{mg}$ SDD twice daily for 9 months is beneficial in the management of chronic periodontitis. Further research is needed to evaluate the added benefits of adjunctive SDD administration in high-risk periodontitis patients. Controversial findings on the use of systemic bisphosphonates to prevent periodontal disease progression and alveolar bone loss have been reported in human studies.

In the future a range of HMTs targeting different aspects of the destructive surge of breakdown episodes in the periodontal tissues are likely to be developed as adjunctive treatments for periodontitis. The further development of these agents will permit dentists to treat specific aspects of the underlying biochemical basis for periodontal disease. The goal is to take full advantage of the treatment response by reducing inflammation and inhibiting destructive processes in the periodontal tissues, which will result in enhanced periodontal stability after conventional periodontal treatments such as scaling and root planing. The dentist is now in the exciting position to be able to combine established treatment strategies with new systemic and local drug treatments for this common, chronic disease.

\section{REFERENCES}

[1]. Maria Emanuel Ryan and Phillip M. Preshaw, Host Modulation, In Carranza's Clinical Periodontology , 10 ${ }^{\text {th }}$ Edition, 275-282, 2007.

[2]. Shalu Bathla, Host Modulatory Therapy, In Periodontics Revisited, $1^{\text {st }}$ Edition, 292-295, 2011

[3]. Genco R, Kornman K, Williams R, Offenbacher S, Zambon J, Ishikawa I, et al. Consensus report periodontal diseases: pathogenesis and microbial factors. Ann Periodontol. 1(1):926-32, 1996.

[4]. Kornman KS. Host modulation as a therapeutic strategy in the treatment of periodontal disease. Clinical infectious diseases, 28(3),520-524, 1999.

[5]. Ciancio S, Ashley R. Safety and efficacy of sub-antimicrobial-dose doxycycline therapy in patients with adult periodontitis, Advances in Dental Research, 12(1):27-31, 1998.

[6]. Golub L, Lee H, Greenwald R, Ryan M, Sorsa T, Salo T, et al. A matrix metalloproteinase inhibitor reduces bone-type collagen degradation fragments and specific collagenases in gingival crevicular fluid during adult periodontitis. Inflammation Research, 46(8):310-319, 1997.

[7]. Lavaneras A, Ramamurthy NS, Heikkilä P, Teronen O, Salo T, Rifkin BR, et al. A combination of a chemically modified doxycycline and a bisphosphonate synergistically inhibits endotoxin-induced periodontal breakdown in rats. Journal of Periodontology, 72 (8):1069-1077, 2001.

[8]. Phillip M, Preshaw, Maria Emanuel Ryan, William V. Giannobile, Host Modulation Agents, In Carranza's Clinical Periodontology , $10^{\text {th }}$ Edition, 813-827, 2007.

[9]. Offenbaceer S, Odle B, Dyke T. The use of crevicular fluid prostaglandin E2 levels as a predictor of periodontal attachment loss. Journal of periodontal research, 21(2):101-112, 1986

[10]. Howell TH, Williams RC. Nonsteroidal antiinflammatory drugs as inhibitors of periodontal disease progression. Critical Reviews in Oral Biology \& Medicine, 4(2):177-196, 1993.

[11]. Nakaya H, Osawa G, Iwasaki N, Cochran D, Kamoi K, Oates T. Effects of bisphosphonate on matrix metalloproteinase enzymes in human periodontal ligament cells. Journal of Periodontology, 71(7):1158-66, 2000.

[12]. Dr. Amit Bhardwaj, Dr. Shalu Verma Bhardwaj, Host Modulation and Host Modulating Agents in Periodontal Therapy, International Journal of Applied Biology and Pharmaceutical Technology, Volume 3, issue -3, pages 103-108, July- Sept 2012.

[13]. Brennan PA, Thomas GJ,Langdon JD, The role of nitric oxide in oral diseases, Arch Oral Biol, 48: 93-100, 2003.

[14]. Zingarelli B, Southan GJ, Gilad, E, O’Connor M, Salzman AL, Szabo C, The inhibitory effects of mercaptoalkylguanidines on cyclooxygenase activity, Brit. J Pharmacol, 120: 357-366, 1997.

[15]. Albandar.JM. Global risk factors and risk indicators for periodontal diseases. Periodontology 2000, 29(1):177-206. 2002

[16]. Offenbaceer S, Odle B, Dyke T. The use of crevicular fluid prostaglandin E2 levels as a predictor of periodontal attachment loss. Journal of periodontal research 21(2):101-112, 1986.

[17]. Trepicchio W, Bozza M, Pednault G, Dorner A. Recombinant human interleukin-11 attenuates the inflammatory response through downregulation of proinflammatory cytokine release and nitric oxide production, Journal of Immunology. 157:3627-3634, 1996.

[18]. Brennan P, Thomas G, Langdon J. The role of nitric oxide in oral diseases. Archives of Oral Biology, 48 (2):93-100. 2003. 